ARTICLE

\title{
Ionic covalent organic framework based electrolyte for fast-response ultra-low voltage electrochemical actuators
}

Fei Yu (D) ${ }^{1}$, Jing-Hao Ciou', Shaohua Chen ${ }^{1}$, Wei Church Poh ${ }^{1}$, Jian Chen ${ }^{1}$, Juntong Chen', Kongcharoen Haruethai ${ }^{1}$, Jian Lv (D ${ }^{1,2}$, Dace Gao ${ }^{1} \&$ Pooi See Lee (i) 1,2凶

Electrically activated soft actuators with large deformability are important for soft robotics but enhancing durability and efficiency of electrochemical actuators is challenging. Herein, we demonstrate that the actuation performance of an ionic two-dimensional covalent-organic framework based electrochemical actuator is improved through the ordered pore structure of opening up efficient ion transport routes. Specifically, the actuator shows a large peak to peak displacement $(9.3 \mathrm{~mm}, \pm 0.5 \mathrm{~V}, 1 \mathrm{~Hz}$ ), a fast-response time to reach equilibrium-bending $(\sim \mathrm{s})$, a correspondingly high bending strain difference $(0.38 \%)$, a broad response frequency $(0.1-20 \mathrm{~Hz})$ and excellent durability ( $>99 \%)$ after 23,000 cycles. The present study ascertains the functionality of soft electrolyte as bionic artificial actuators while providing ideas for expanding the limits in applications for robots.

\footnotetext{
${ }^{1}$ School of Materials Science and Engineering, Nanyang Technological University, Singapore 639798, Singapore. ${ }^{2}$ Singapore-HUJ Alliance for Research and Enterprise (SHARE), Nanomaterials for Energy and Water Nexus (NEW), Campus for Research Excellence and Technological Enterprise (CREATE),

Singapore 639798, Singapore. ${ }^{凶}$ email: pslee@ntu.edu.sg
} 
B ionic artificial muscles that exhibit reversible motion responses upon external stimuli (heat, $\mathrm{pH}$, humidity, salt, solvent, electric or magnetic field, and light) have made remarkable progress over the past decades ${ }^{1-13}$. Because of their flexibility ${ }^{14,15}$, low cost, low pollution, no noise, lightweight, low activation voltage, versatile deformations, and rapid response ${ }^{16}$, electroactive polymer (EAP) actuators have emerged as versatile materials for high-efficiency micro- and macro-artificial muscles and soft robotic systems ${ }^{17,18}$, biomedical devices ${ }^{19}$, and biomimetic flying insects ${ }^{20}$. In particular, ionic-polymer metal composites (IPMCs) actuators are positioned as promising candidates due to their decent bending actuation at ultralow voltages and are among the most promising EAP materials for artificial muscle constructed with ionic-conductive electrolytes, mobile molten ionic salts, and sandwiches with metallic conductors ${ }^{21-25}$. The deformation of the IPMC actuator is based on the volume or pressure gradient of the actuator, reversible ion insertion, and deintercalated under the applied electrical stimulation ${ }^{26}$. Over the past decades, various types of actuators have been widely explored ${ }^{27-30}$. However, the long-term challenge remains in designing and manufacturing actuators with high electromechanical conversion efficiency, stronger mechanical output force, fast response, large stress/strain density, and operational durability in the air, as the present IPMC actuation performances cannot meet the demands for practical applications ${ }^{31-33}$.

Ionic covalent organic frameworks (iCOFs), as one of the rapidly developing subclasses of crystalline materials, have been developed because of their regular porosity architectures and high ionic conductivities ${ }^{34-38}$. The distinctive structural and chemical characteristics and high Brunauer-Emmett-Teller (BET) surface area offer appealing benefits for increased ion transport via the frameworks. These features can usually be used in ionconducting $34,39-41$, proton conduction ${ }^{35,36}$, iodine capture ${ }^{42}$, catalysis $^{43}$, fuel cell ${ }^{44}$, electro/photochromic ${ }^{45-47}$, and gas separation ${ }^{48}$. The tubular one-dimensional (1D) hole, created between the two-dimensional (2D) COF layers, may provide effective ion-transport pathways that are associated with the impact of the confinement and relative ions and pore size. To the best of our knowledge, the potential of high-performance twodimensional ionic COF-based electrolytes in electrochemical actuators has largely remained unexplored.

In this work, a two-dimensional (2D) ionic COF (COF-DT$\mathrm{SO}_{3} \mathrm{Na}$ ) with 1,3,5-tris(4-aminophenyl)benzene (TAB) and 2,5dihydroxyterephthalaldehyde (DHA) building blocks is prepared (Fig. 1) and employed as an active element in electrochemical soft actuators. The COF-DT-SO ${ }_{3} \mathrm{Na}$-based electrolyte actuator has a high actuation displacement $(9.3 \mathrm{~mm}, 1 \mathrm{~Hz})$, a bending-strain difference of $0.38 \%(1 \mathrm{~Hz})$, a strong resonance displacement $(\sim 12.1 \mathrm{~mm}, 8 \mathrm{~Hz})$, a rapid time to reach equilibrium-bending motion $(\sim 1 \mathrm{~s})$, a wide-frequency $(0.1-20 \mathrm{~Hz})$ response, and longterm durability ( $>23,000$ cycles) under continuous electrical stimuli in the air with negligible degradation in actuation performance under $\pm 0.5 \mathrm{~V}$ at a frequency of $0.1 \mathrm{~Hz}$. The increased actuation performance is due to both the oriented porous structure and efficient transfer routes for rapid ion transport while facilitating significantly high diffusion rates ${ }^{34,39-41}$. In addition, the excellent voltage-driven deformation of COF-DT- $\mathrm{SO}_{3} \mathrm{Na}$ actuators can mimic biological motions, such as flowering, tendril curling, and butterfly high-frequency flapping, which makes COF-DT- $\mathrm{SO}_{3} \mathrm{Na}$ actuators as a promising candidate for future biomimetic robots or soft biomedical end effectors.

\section{Results}

COF design and synthesis. The building blocks of TAB and DHA were synthesized based on the previous methods with slight modifications (Supplementary Methods) ${ }^{49}$. The 2D COF (COFDT) was synthesized as follows: TAB and DHA were first dissolved in the solvent of $n$-butanol:1,2-dichlorobenzene:acetic acid $(6 \mathrm{M})(v / v / v=5: 5: 1)$, then the mixture was ultrasonicated for 30 minutes to generate a red slurry, followed by freezing in liquid nitrogen at $77 \mathrm{~K}$, degassing through four freeze-pump-thaw cycles, and sealed in a Schlenk flask. The mixture was heated at $120^{\circ} \mathrm{C}$ for $96 \mathrm{~h}$ and yielded red precipitates of COF-DT. Then, the product reacted with 1,3-propanesultone at $110^{\circ} \mathrm{C}$ in toluene for $6 \mathrm{~h}$, allowing the sulfonate ester to react with the $\mathrm{OH}$ functional group of COF-DT. This results in the alkyl chain with the terminal sulfonic acid functional group fixed inside the channel, giving dark red precipitates of COF-DT- $\mathrm{SO}_{3} \mathrm{H}$. In the end, the COF-DT- $\mathrm{SO}_{3} \mathrm{H}$ reacted with $\mathrm{NaOH}(1 \mathrm{M})$ at room temperature for ion-exchange reaction with the sulfonic acid functional groups and yielded the final product as COF-DT-SO ${ }_{3} \mathrm{Na}$ (Fig. 2).

Structure characterization. The as-synthesized COF-DT powder $\mathrm{X}$-ray diffraction (PXRD) pattern displays several discrete Bragg reflections with $2 \theta$ roughly at $2.8,4.9,5.6$, and $7.4^{\circ}$, which are compatible with ( $\left.\begin{array}{lll}1 & 0 & 0\end{array}\right),\left(\begin{array}{lll}1 & 1 & 0\end{array}\right),\left(\begin{array}{lll}2 & 0 & 0\end{array}\right)$, and (llll $\left.\begin{array}{lll}1 & 2 & 0\end{array}\right)$ faces in the modeled structure. In addition, Pawley refinement was utilized to uncover the $P 6 / \mathrm{m}$ space group and a unit cell with $a=38.19$, $b=38.19, c=3.48 \AA$, and $\gamma=120^{\circ}$ with $R_{\mathrm{wp}}=4.33 \%$ and $R_{\mathrm{p}}=$ $3.34 \%$. According to their difference, there is a good agreement between the experimental data and the refined pattern (Fig. 3a). The diffraction patterns of COF-DT-SO ${ }_{3} \mathrm{H}$ and $\mathrm{COF}-\mathrm{DT}-\mathrm{SO}_{3} \mathrm{Na}$ are in good match with the diffraction pattern of COF-DT (Fig. 3b), indicating that the framework remains stable after postsynthetic modification. Transmission electron microscope (TEM) samples were prepared by immersing COF-DT powder in ethanol and sonicating it at a power of $100 \mathrm{~W}$ and a frequency of $37 \mathrm{kHz}$ for 60 minutes. The TEM image of the sample (Fig. 3c) clearly shows parallel lines with an adjacent distance of $\sim 3.4 \AA$, which is attributed to the stacking structure around the $c$-axis of the COFDT. Furthermore, the attenuated total reflection Fourier transform-infrared (ATR FT-IR) spectra of COF-DT- $\mathrm{SO}_{3} \mathrm{H}$ and COF-DT-SO ${ }_{3} \mathrm{Na}$ show two additional peaks at 1034 and $1042 \mathrm{~cm}^{-1}$, corresponding to $\mathrm{S}=\mathrm{O}$ stretching vibration, which verified the effective integration of sulfonic groups into COF-DT (Supplementary Fig. 3) ${ }^{49}$. The energy-dispersive X-ray (EDX) spectroscopy showed homogeneously dispersed emissions from $\mathrm{C}, \mathrm{N}$, and $\mathrm{O}$ for COF-DT, from $\mathrm{C}, \mathrm{N}, \mathrm{O}$, and $\mathrm{S}$ for COF-DT$\mathrm{SO}_{3} \mathrm{H}$, and from $\mathrm{C}, \mathrm{N}, \mathrm{O}, \mathrm{S}$, and $\mathrm{Na}$ for COF-DT-SO ${ }_{3} \mathrm{Na}$ (Supplementary Fig. 4).

Brunauer-Emmett-Teller (BET) study indicates that COF-DT has a reversible adsorption isotherm of $\mathrm{N}_{2}$ at $77 \mathrm{~K}$, resulting in a BET surface area of $1287 \mathrm{~m}^{2} \mathrm{~g}^{-1}$ with a pore diameter of $\sim 3.2 \mathrm{~nm}$ by using non-local density functional theory (NLDFT) modeling. For the post-synthesized compounds of COF-DT-SO ${ }_{3} \mathrm{H}$ and COF-DT$\mathrm{SO}_{3} \mathrm{Na}$, the BET surface areas are 470 and $381 \mathrm{~m}^{2} \mathrm{~g}^{-1}$, with the pore diameters of $\sim 2.5$ and $2.4 \mathrm{~nm}$, respectively (Fig. 3d and Supplementary Fig. 5). These results demonstrate that the effects on the crystallinity and pore structure of the original material are negligible by post-synthetic modification. In addition, these nanopores can provide necessary space and act as nanoreactors to transfer oppositely charged ions without stress during the switching of AC input signals in the presence of available electrolytes.

Due to the large number of sulfonated groups neatly arranged in one-dimensional nanoporous channels, COF-DT- $\mathrm{SO}_{3} \mathrm{Na}$ is expected to be a highly ionic conductive material ${ }^{37,38}$. To enhance ion migration, $10 \mu \mathrm{L}$ of EC/DMC $(v / v=1: 1)$ mixed solvent was added to COF-DT- $\mathrm{SO}_{3} \mathrm{Na}$ as a plasticizer. Temperaturedependent $\left(20-80^{\circ} \mathrm{C}\right)$ electrochemical impedance spectroscopy (EIS) of COF-DT-SO ${ }_{3} \mathrm{Na}$ revealed a superior conduction 


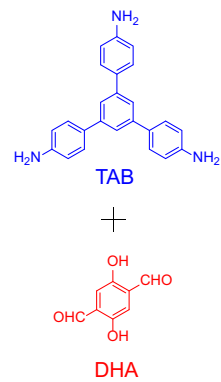

o-dichlorobenzene

$120^{\circ} \mathrm{C}$
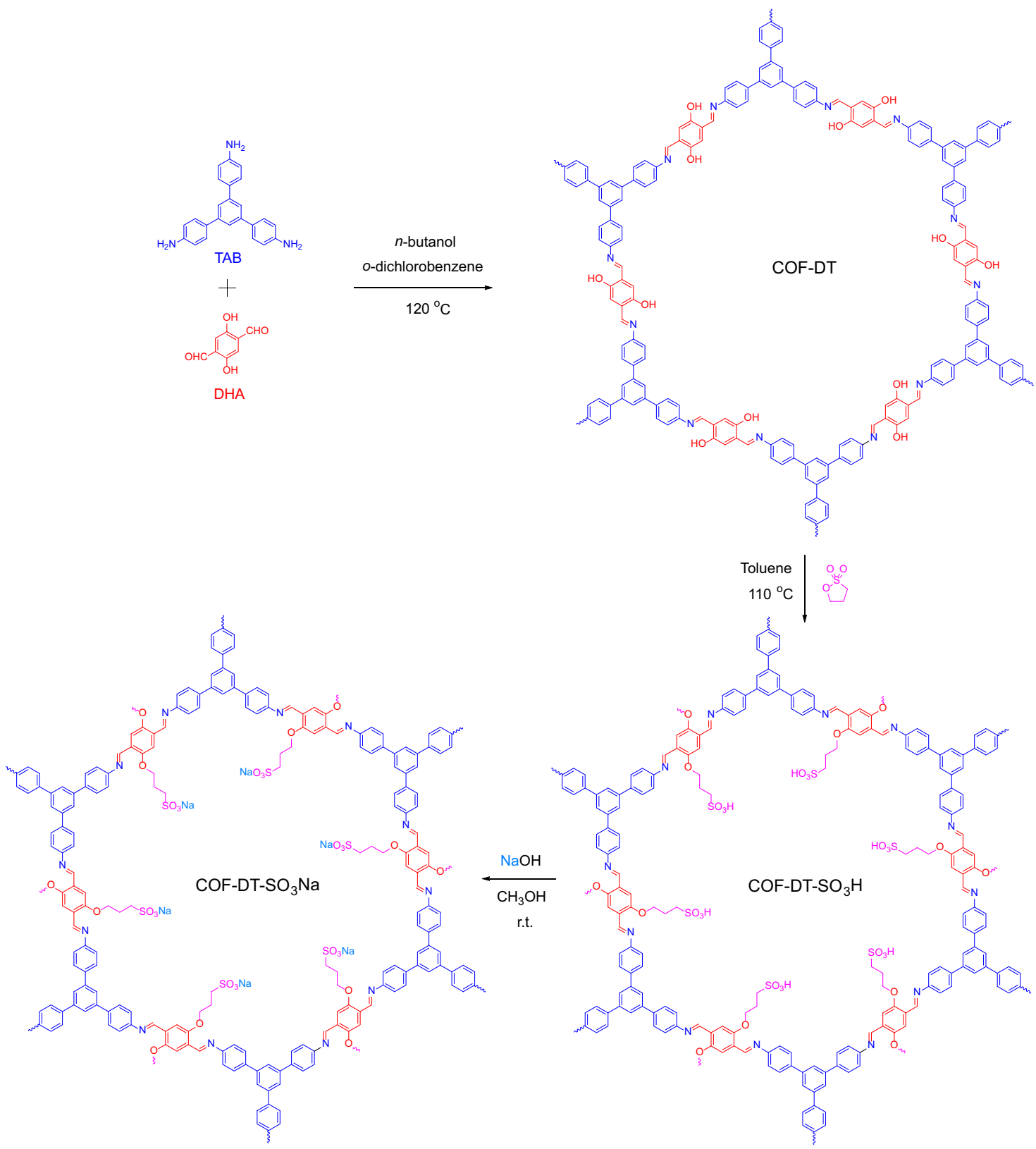

Fig. 1 Synthesis of ionic COF. Synthetic route of ionic COF-DT-SO $\mathrm{Na}$.

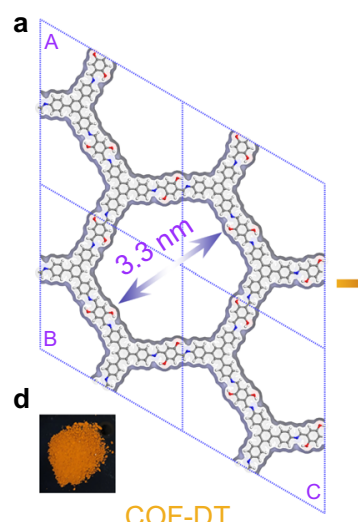

b
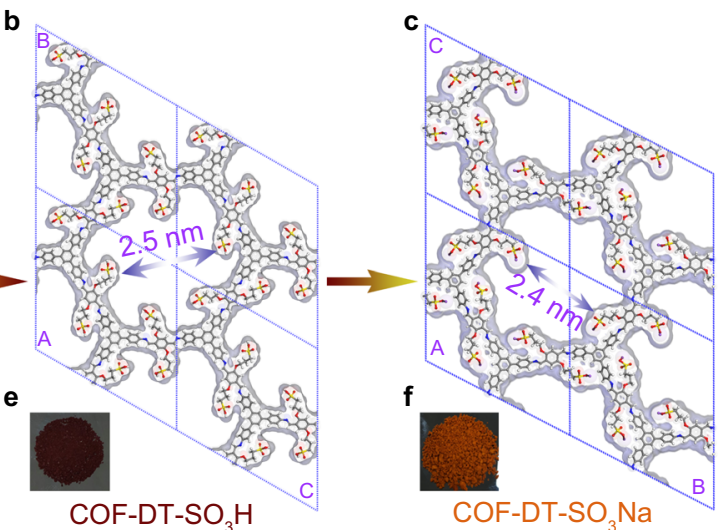

Fig. 2 Structural aspects of COF. Simulated structure of a COF-DT, b COF-DT-SO ${ }_{3} \mathrm{H}$, and $\mathbf{c}$ COF-DT-SO ${ }_{3} \mathrm{Na}$ (Colors: blue-N, gray-C, red-O, yellow-S, pink- $\mathrm{Na}$, white-H) with the pore sizes of $3.3,2.5$, and $2.4 \mathrm{~nm}$, respectively. The powder-sample images under visible light of $\mathbf{d}$ COF-DT, e COF-DT-SO ${ }_{3} \mathrm{H}$, and $\mathbf{f} \mathrm{COF}-\mathrm{DT}-\mathrm{SO}_{3} \mathrm{Na}$. 

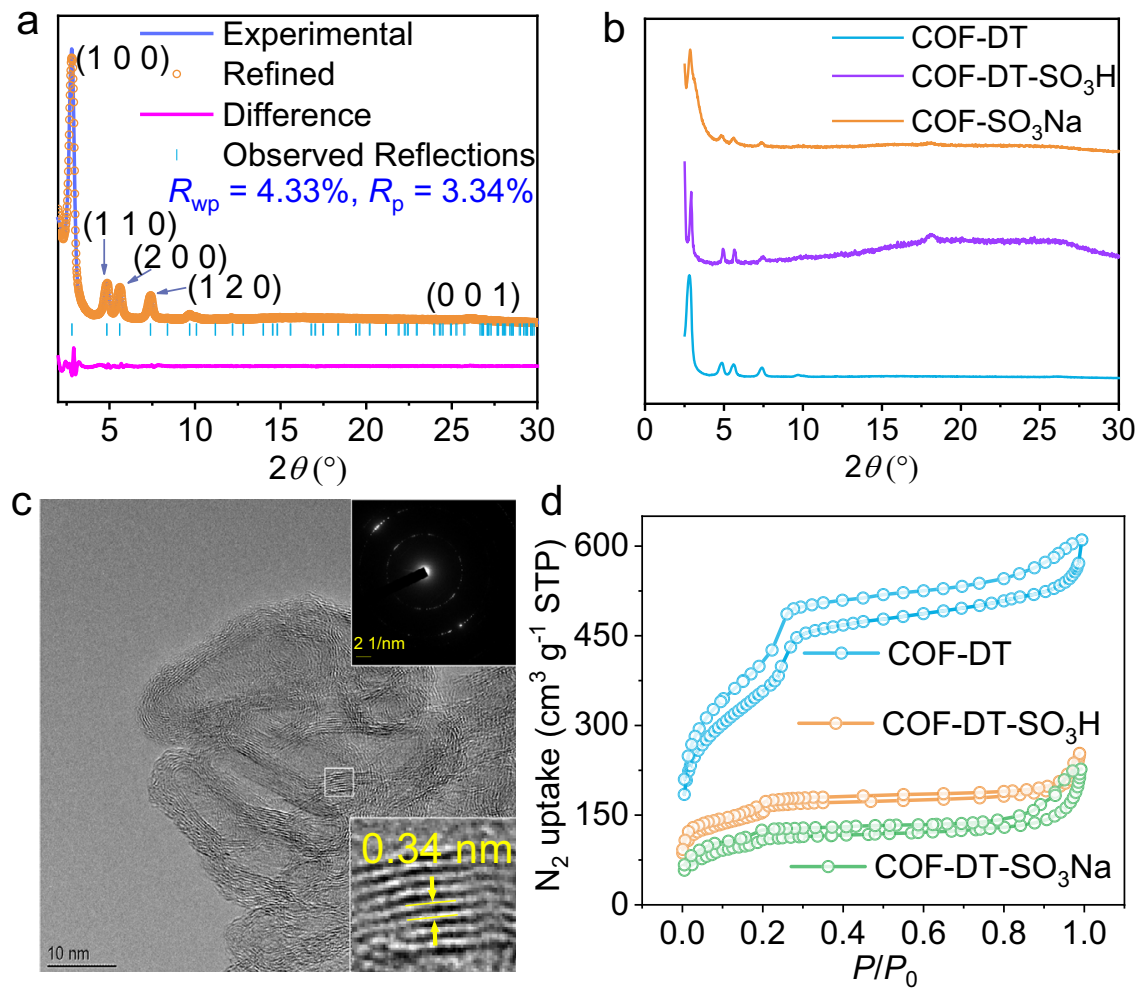

Fig. 3 Characterizations of COF-DT, COF-DT-SO ${ }_{3} \mathbf{H}$, and COF-DT-SO ${ }_{3} \mathbf{N a}$. a The experimental powder X-ray diffraction of COF-DT (blue line), the Pawley refined plot (yellow circle), and the difference between experimental and refined plot (purple line). $\mathbf{b}$ Comparison of experimental powder $\mathrm{X}$-ray diffraction pattern of COF-DT (cyan line), COF-DT-SO ${ }_{3} \mathrm{H}$ (green line), and COF-DT- $\mathrm{SO}_{3} \mathrm{Na}$ (red line). c HR-TEM image of COF-DT. $\mathbf{d}$ Porous properties: $\mathrm{N}_{2}$ sorption isotherm of COF-DT (cyan circle line), COF-DT-SO ${ }_{3} \mathrm{H}$ (orange circle line), and COF-DT- $\mathrm{SO}_{3} \mathrm{Na}$ (green circle line).

performance of the ionic COF pellet. The conductivities (Supplementary Equation 1) were calculated as 13.5, 16.5, 19.4, 23.8, 31.6, 38.4, and $45.2 \mathrm{mS} \mathrm{cm}^{-1}$ at 293, 303, 313, 323, 333, 343, and $353 \mathrm{~K}$, respectively (Fig. 4a). Arrhenius fitting result shows that the activation energy $\left(E_{\mathrm{a}}\right)$ of COF-DT- $\mathrm{SO}_{3} \mathrm{Na}$ is $0.18 \mathrm{eV}$ (Fig. 4b). To evaluate the potential electronic conductivity of COF-DT- $\mathrm{SO}_{3} \mathrm{Na}$, the impedance of the geometrically equivalent COF-DT and COF-DT- $\mathrm{SO}_{3} \mathrm{H}$ without adding the EC/DMC mixed solvent $\left(25^{\circ} \mathrm{C}, 30 \% \mathrm{RH}\right)$ was also examined. The results reveal that both materials were electric insulators (Supplementary Figs. 6 and 7).

Actuation performance. To explore the functional characteristics of COF-DT- $\mathrm{SO}_{3} \mathrm{Na}$ electrolyte in practical applications, a mixture of COF-DT-SO ${ }_{3} \mathrm{Na}$ and EC/DMC/NMP $(v / v / v=1: 1: 1)$ ionic layer was used as a quasi-solid electrolyte between PEDOT:PSS electrodes (Fig. 4c). The cross-sectional morphology of the assembled soft actuator shows an interlayer adhesion between the PEDOT:PSS electrodes and the COF-DT- $\mathrm{SO}_{3} \mathrm{Na}$ electrolyte, which promotes ion diffusion and significantly reduces iontransport resistance (Fig. 4c). The CV curve of the device was measured under $\pm 0.5 \mathrm{~V}$ at a scan rate from 100 to $1000 \mathrm{mV} \mathrm{s}^{-1}$ (Fig. 4d). The non-Faradaic process that occurs at the electrode/ electrolyte interface is primarily responsible for the properties of the electric double-layer capacitor. The reversible CV curve gives an area capacity of $125.2 \mathrm{mF} \mathrm{cm}^{-2}$ at scan rate of $100 \mathrm{mV} \mathrm{s}^{-1}$. The actuation performance of a COF-DT- $\mathrm{SO}_{3} \mathrm{Na}$ soft actuator with a strip shape (width $4 \mathrm{~mm}$, length $20 \mathrm{~mm}$, and thickness 60 $\mu \mathrm{m})$ was studied under a square-wave alternating-current (AC) voltage of $\pm 0.5 \mathrm{~V}$ at $0.1 \mathrm{~Hz}$, showing that the peak-to-peak displacement $(\delta)$ reaches a maximum value of $9.6 \mathrm{~mm}$. In addition, due to the expansion and contraction of the PEDOT:PSS electrode during the $\mathrm{COF}-\mathrm{DT}-\mathrm{SO}_{3} \mathrm{Na}$ ion-intercalation process, reversible deformation was observed in both directions (Fig. $4 \mathrm{e}$ and Supplementary Movie 1).

The displacements $(\delta)$ shown as a function of frequencies under $0.1-20 \mathrm{~Hz}$ at $\pm 0.5 \mathrm{~V}$ were also evaluated (Fig. 5a). The actuator reaches the highest displacement $(\sim 9.6 \mathrm{~mm})$ at the frequency of $0.1 \mathrm{~Hz}$. From $1 \mathrm{~Hz}$ to $0.1 \mathrm{~Hz}$, the maximum displacements of the actuator are almost the same $(\sim 9.3 \mathrm{~mm}$, $1 \mathrm{~Hz})$, indicating that the actuator can quickly $(\sim 1 \mathrm{~s})$ reach the equilibrium-bending motion (Supplementary Fig. 8). Such fast equilibrium time can be attributed to the nanopores in the COFDT- $\mathrm{SO}_{3} \mathrm{Na}$ to which they provide the necessary space to transfer the oppositely charged ions without pressure during the switching process of the AC input signal. The peak-to-peak displacement decreases gradually with increasing frequency at the range of 1-5 Hz. Furthermore, when the frequency reaches an appropriate value $(\sim 8 \mathrm{~Hz})$, the soft actuator will resonate strongly, and the swing amplitude will jump to the maximum displacement $(\sim 12.1 \mathrm{~mm})$. Moreover, in the range of $9-20 \mathrm{~Hz}$, as the operating frequency increases, the displacement gradually decreases, while above $20 \mathrm{~Hz}$, the actuation performance almost disappears (Fig. 5a and Supplementary Movie 1).

Such excellent actuation performance encourages us to further explore the COF-DT- $\mathrm{SO}_{3} \mathrm{Na}$ actuator in the air at the lower voltage. The maximum displacement becomes 6.7 and $2.7 \mathrm{~mm}$ under voltages of \pm 0.3 and $\pm 0.1 \mathrm{~V}$ at $0.1 \mathrm{~Hz}$, respectively. The resonance behavior can be also determined near the frequency of $8 \mathrm{~Hz}$, resulting in the displacement of 5.8 and $2.4 \mathrm{~mm}$ at \pm 0.3 and $\pm 0.1 \mathrm{~V}$, respectively. Moreover, in the range of $9-20 \mathrm{~Hz}$, as the operating frequency increases, the displacement gradually decreases, while above $20 \mathrm{~Hz}$, the actuation performance almost disappears (Fig. 5b). 

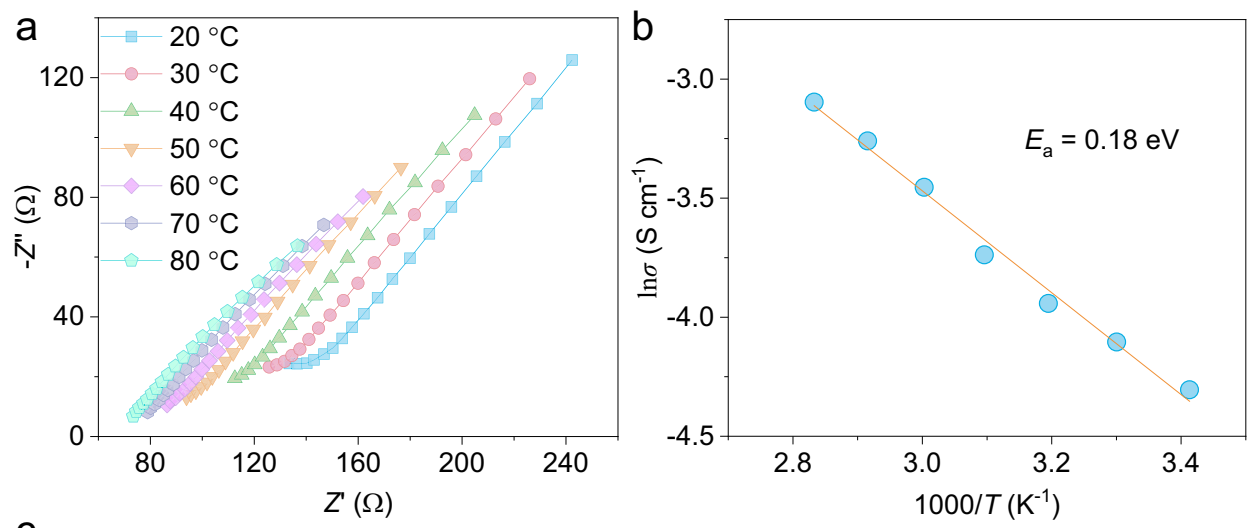

C
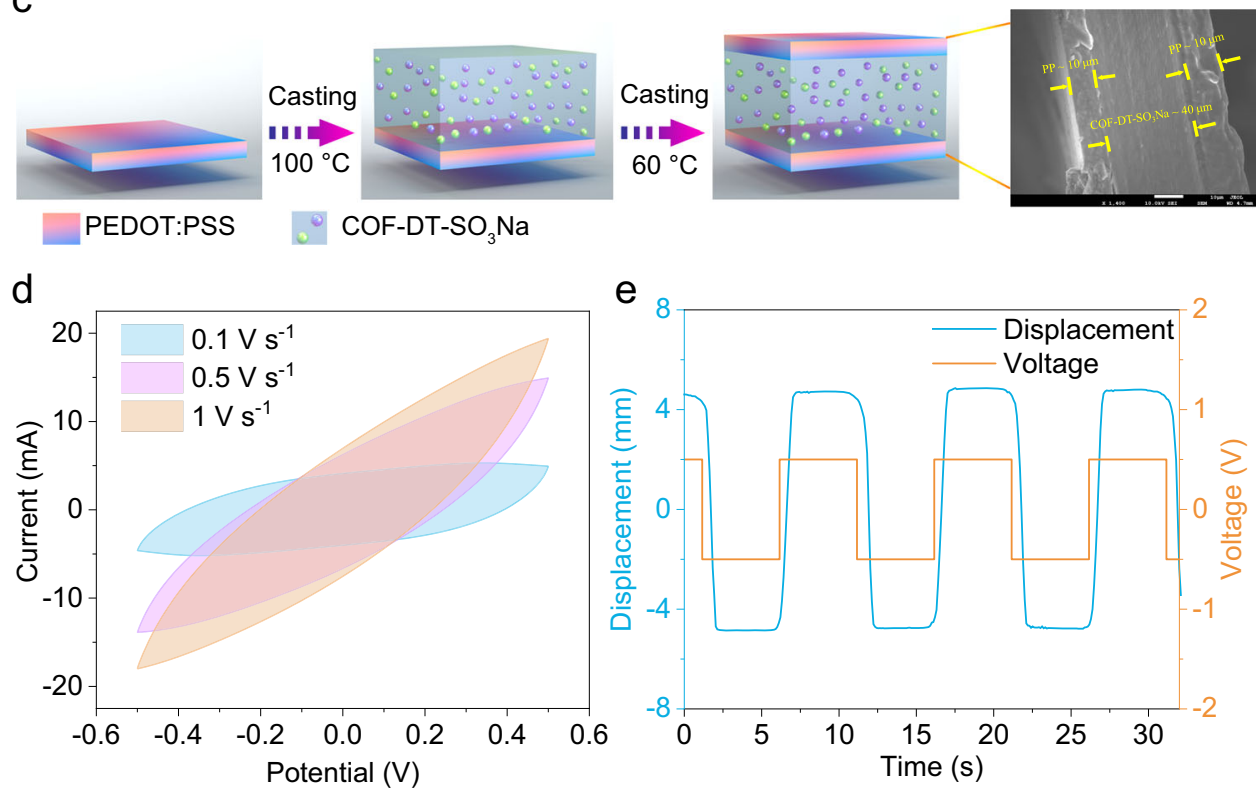

Fig. 4 Characterizations of COF-DT-SO ${ }_{3} \mathrm{Na}$-based actuator. a EIS plot of COF-DT-SO ${ }_{3} \mathrm{Na}$. In all, $20^{\circ} \mathrm{C}$ (blue quadrilateral line), $30^{\circ} \mathrm{C}$ (red circle line), $40^{\circ} \mathrm{C}$ (green upward triangle line), $50^{\circ} \mathrm{C}$ (orange downward triangle line), $60^{\circ} \mathrm{C}$ (pink quadrilateral line), $70^{\circ} \mathrm{C}$ (cerulean-blue hexagon line), $80^{\circ} \mathrm{C}$ (cyan pentagon line). b Arrhenius fitting (orange line) of In (conductivity) versus scaled inverse temperature, experimental data (blue circle). c The process of fabricating DT-COF-SO ${ }_{3} \mathrm{Na}$-based soft actuators. $\mathbf{d}$ The $\mathrm{CV}$ curves of the COF-DT- $\mathrm{SO}_{3} \mathrm{Na}$ based soft actuator at various scan rates, $0.1 \mathrm{~V} \mathrm{~s}{ }^{-1}$ (blue), $0.5 \mathrm{~V} \mathrm{~s}^{-1}$ (red), and $1 \mathrm{~V} \mathrm{~s}^{-1}$ (orange). e The displacement (blue line) of COF-DT-SO ${ }_{3} \mathrm{Na}$-based soft actuator under an $\mathrm{AC}$ square-wave voltage (orange line) of $\pm 0.5 \mathrm{~V}$ at $0.1 \mathrm{~Hz}$.

The actuator produces $0.39 \%, 0.30 \%$, and $0.14 \%$ bendingstrain differences (\%) (Supplementary Equation 2) under \pm 0.5 , \pm 0.3 , and $\pm 0.1 \mathrm{~V}$ at $0.1 \mathrm{~Hz}$, respectively (Fig. $5 \mathrm{c}$ ). Specifically, the COF-DT- $\mathrm{SO}_{3} \mathrm{Na}$ actuator maintains a relatively large displacement of $0.4 \mathrm{~mm}$ and a bending-strain difference of $0.016 \%$ at $20 \mathrm{~Hz}$. As a comparison, the Nafion-based actuator has no actuation performance at the frequency above $2 \mathrm{~Hz}$ and has a slower equilibrium time (>10 s) (Supplementary Fig. 9). In addition, one of the important aspects of the design of artificial bioinspired actuation systems is the blocking force. Under an alternating-current input voltage of $0.5 \mathrm{~V}$ at $0.1 \mathrm{~Hz}$, the blocking force generated $1.2 \mathrm{mN}$ at the tip of the actuator. In addition, the long-term stability of the actuator was also evaluated under $\pm 0.5 \mathrm{~V}$ at $0.1 \mathrm{~Hz}$ (Fig. $5 \mathrm{~d}$ ). The actuator has negligible degradation after more than 23,000 cycles under continuous electric stimuli. A comparison of the bending performance of the state-of-the-art ionic soft actuators with that of COF-DT- $\mathrm{SO}_{3} \mathrm{Na}$ actuator is listed in Supplementary Table 4. It demonstrates that COF-DT- $\mathrm{SO}_{3} \mathrm{Na}$ actuator has competitive performance to other materials under $\pm 0.5 \mathrm{~V}, 1 \mathrm{~Hz}$. A weak butterfly robot was constructed with COF-DT-SO ${ }_{3} \mathrm{Na}$ actuators as the artificial muscles for wing movement to illustrate the actuator soft robotic capability. The butterfly was adhering to a glass rod and connected to the power supply. The wings of the butterfly soft robot started flapping upon applying $\mathrm{a} \pm 0.5 \mathrm{~V}$ under $4-$ and $5-\mathrm{Hz}$ input electrical stimulus (Supplementary Movie 2). These results confirm that highly oriented and integrated ionic COF-DT- $\mathrm{SO}_{3} \mathrm{Na}$ electrolyte provides efficient transfer pathways endowing the electric stimulus with high ionic conductivity, and facilitates the rapid electrolyte migration between the pores, thus giving rise to its fastresponsive actuation nature and long-term stability.

\section{Discussion}

We developed an ionic COF-DT- $\mathrm{SO}_{3} \mathrm{Na}$ electrolyte with a highly oriented porous structure for electrochemical soft actuators operating under ultralow electric stimulus $(\leq \pm 0.5 \mathrm{~V})$. The COFDT- $\mathrm{SO}_{3} \mathrm{Na}$-based ionic soft actuator demonstrates high actuation performance, with large actuation displacement $(\sim 9.6 \mathrm{~mm})$, a large strain difference of $0.39 \%$, a fast-attain equilibrium-bending motion $(\sim 1 \mathrm{~s})$, a broadband frequency response of $0.1-20 \mathrm{~Hz}$, and 

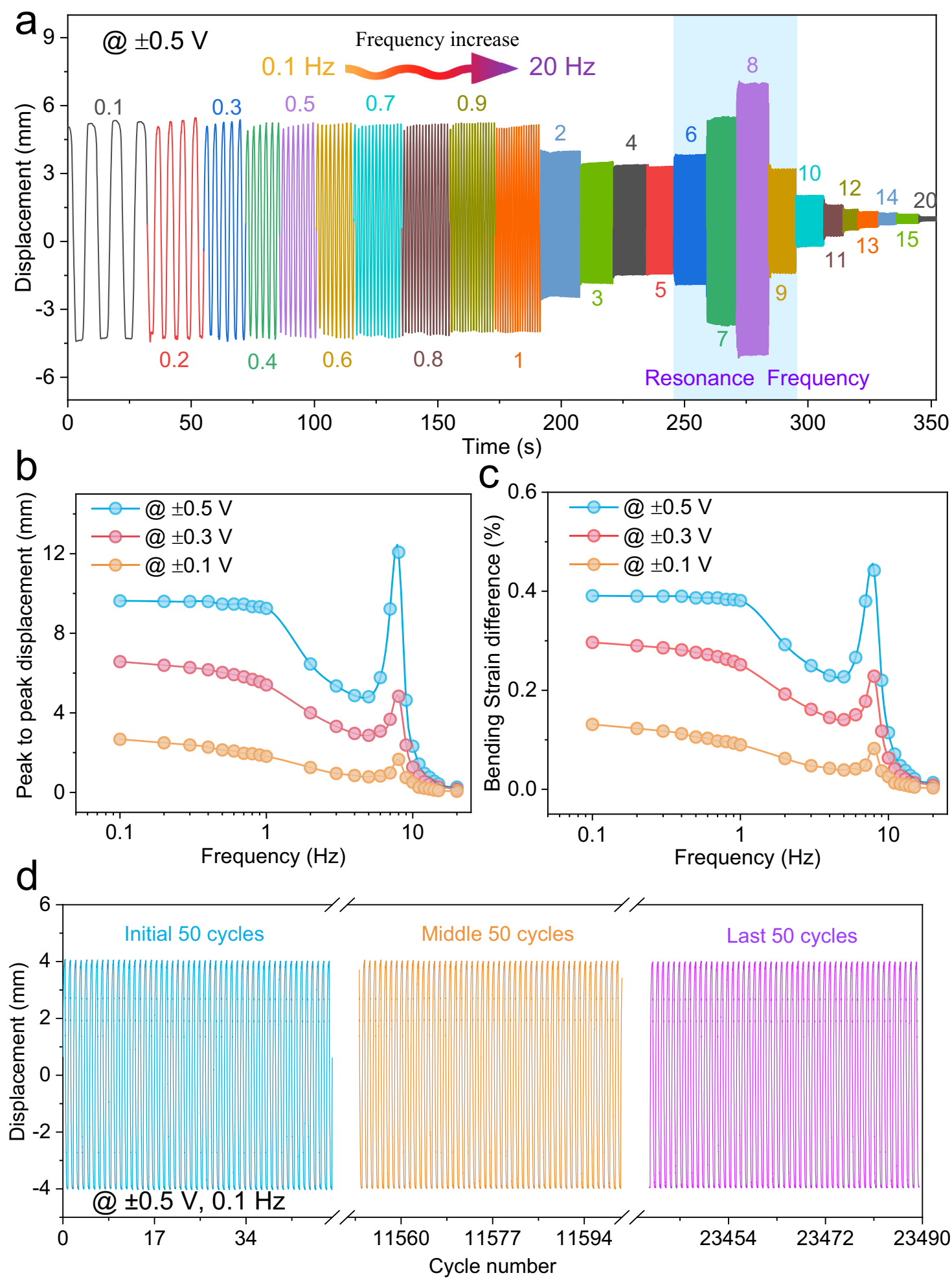

Fig. 5 The actuation performance of the COF-DT-SO $\mathbf{S}_{3} \mathbf{N a}$-based actuator. a The peak to peak displacement under the frequency of $0.1-20 \mathrm{~Hz}$ at $\pm 0.5 \mathrm{~V}$. b Peak-to-peak displacement under the frequency of $0.1-20 \mathrm{~Hz}$ at $\pm 0.5 \mathrm{~V}$ (blue circle line), $\pm 0.3 \mathrm{~V}$ (red circle line), $\pm 0.1 \mathrm{~V}$ (orange circle line), respectively. c Bending-strain difference (\%) under the frequency of $0.1-20 \mathrm{~Hz}$ at $\pm 0.5 \mathrm{~V}$ (blue circle line), $\pm 0.3 \mathrm{~V}$ (red circle line), and $\pm 0.1 \mathrm{~V}$ (orange circle line), respectively. $\mathbf{d}$ Long-term stability of the soft actuator under $\pm 0.5 \mathrm{~V}$ at $0.1 \mathrm{~Hz}$ in the air, initial 50 cycles (blue line), middle 50 cycles (orange line), and last 50 cycles (purple line).

long-term durability in the air $(>23,000$ cycles), thanks to its ordered pores $(\sim 2.4 \mathrm{~nm})$, large specific surface area $\left(381 \mathrm{~m}^{2} \mathrm{~g}^{-1}\right)$, and high ionic conductivity $\left(13.5 \mathrm{mS} \mathrm{cm}^{-1}\right)$. The hierarchical porosity and distinctive COF-DT- $\mathrm{SO}_{3} \mathrm{Na}$ electrolyte structure offer efficient transmission and extensive diffusion channels to facilitate mass transportation and ion intercalation/de-intercalation of solid electrical elements in electrodes. We thereby illustrate utilizing a $2 \mathrm{D}$ ionically conducting COF-DT- $\mathrm{SO}_{3} \mathrm{Na}$ design, 
with excellent carrier accessibility that provides excellent actuation performance, further encouraging the development of such materials in the application of actuation. We anticipate that the method described in this study will open up possibilities for the efficient development of high-performance actuator materials by providing a basis for multifunctional actuators.

\section{Methods}

Synthesis of COF-DT. About $1.2 \mathrm{~mL}$ of a 5:5:1 $(v / v / v)$ solution of 1,2-dichlorobenzene/ $n$-butylalcohol/ $6 \mathrm{M}$ aqueous acetic acid was added to a combination of 2,5-dimethoxyterephthalaldehyde (DHA, $24.9 \mathrm{mg}, 0.15 \mathrm{mmol}$ ) and 1,3,5-tris(4aminophenyl)benzene (TAB, $35.1 \mathrm{mg}, 0.1 \mathrm{mmol})$ in a Schlenk tube $(10 \mathrm{~mL})$. After 30 min of sonication, it was flash-frozen in liquid $\mathrm{N}_{2}$ at $77 \mathrm{~K}$, evacuated, and sealed under vacuum. The mixture was heated at $120^{\circ} \mathrm{C}$ for $96 \mathrm{~h}$, yielding an orange precipitate that was separated by filtering and washed with anhydrous tetrahydrofuran for 7 days using Soxhlet extraction. Yielding denoted as COF-DT. Yield: $48.1 \mathrm{mg}(80 \%)$.

Synthesis of COF-DT-SO 3 H. COF-DT $\left(500 \mathrm{mg}\right.$ ) was evacuated at $100{ }^{\circ} \mathrm{C}$ for $6 \mathrm{~h}$, then, cooled to room temperature, and added a mixture of 1,3-propanesultone $(0.5 \mathrm{~mL})$ in $30 \mathrm{~mL}$ of dry toluene. The resulting mixture was reflux for another $6 \mathrm{~h}$ under $\mathrm{N}_{2}$ atmosphere. After being cooled down, the solid was filtered, washed thoroughly with toluene, and dried at $60{ }^{\circ} \mathrm{C}$ overnight under vacuum drying oven, yielding a red powder that was denoted as COF-DT- $\mathrm{SO}_{3} \mathrm{H}$.

Synthesis of COF-DT-SO ${ }_{3} \mathrm{Na}$. Methanol $(20 \mathrm{~mL})$ was added to a combination of COF-DT-SO ${ }_{3} \mathrm{H}(200 \mathrm{mg})$ and $1 \mathrm{M} \mathrm{NaOH}(1 \mathrm{~mL})$ in a $50 \mathrm{~mL}$ Schlenk flask under $\mathrm{N}_{2}$. COF-DT-SO $\mathrm{N}_{3} \mathrm{Na}$ was obtained after stirring at room temperature for 6 hours, filtering, and washing with methanol, and drying under vacuum at $50{ }^{\circ} \mathrm{C}$.

\section{Fabrication of the COF-DT-SO $\mathrm{S}_{3} \mathrm{Na}$-based electrochemical actuators. About} $5 \mathrm{wt} \%$ of DMSO was added into the aqueous PEDOT:PSS commercial solution. Layer-by-layer sandwiched PEDOT:PSS-COF-DT-SO ${ }_{3} \mathrm{Na}-\mathrm{PEDOT}$ :PSS membrane was fabricated by the drop-casting. Specifically, the PEDOT:PSS solution was casted on a glass slide uniformly under $60^{\circ} \mathrm{C}$ for $30 \mathrm{~min}$. Then COF-DT$\mathrm{SO}_{3} \mathrm{Na}$ and EC:DMC:NMP $(v / v / v=1: 1: 1)$ solution $\left(10 \mathrm{mg} \mathrm{mL}^{-1}\right)$ was dropped on the precast PEDOT:PSS membrane and dried at $100^{\circ} \mathrm{C}$ for $60 \mathrm{~min}$ (Supplementary Fig. 10). The PEDOT:PSS solution was dropped on the PEDOT:PSS-COF-DT-SO ${ }_{3} \mathrm{Na}$ uniformly under $60^{\circ} \mathrm{C}$ for $30 \mathrm{~min}$ to get a composite actuator.

Structure characterization. Powder X-ray diffraction (PXRD) patterns were conducted on a PANalytical X'Pert Pro MPD diffractometer using $\mathrm{Cu} \mathrm{K} \alpha$ radiation $(\lambda=1.5406 \AA)$ and operating at $40 \mathrm{kV}$ and $40 \mathrm{~mA}$ between 2 and $30^{\circ}(2 \theta)$. TEM was performed with a JEM-2100 (JEOL Ltd., Japan) with an accelerating voltage of $200 \mathrm{kV}$.

\section{Data availability}

The data that support the findings of this study are available within the article and Supplementary Information files, or available from the corresponding authors on request. Source data are provided with this paper.

Received: 31 August 2021; Accepted: 16 December 2021;

Published online: 19 January 2022

\section{References}

1. Ahn, S. K., Kasi, R. M., Kim, S. C., Sharma, N. \& Zhou, Y. Stimuli-responsive polymer gels. Soft Matter 4, 1151-1157 (2008).

2. Okuzaki, H., Kuwabara, T., Funasaka, K. \& Saido, T. Humidity-sensitive polypyrrole films for electro-active polymer actuators. Adv. Funct. Mater. 23, 4400-4407 (2013).

3. Bruns, C. J. \& Stoddart, J. F. Rotaxane-based molecular muscles. Acc. Chem. Res. 47, 2186-2199 (2014).

4. Erbas-Cakmak, S., Leigh, D. A., McTernan, C. T. \& Nussbaumer, A. L. Artificial molecular machines. Chem. Rev. 115, 10081-10206 (2015).

5. Rus, D. \& Tolley, M. T. Design, fabrication and control of soft robots. Nature 521, 467-475 (2015)

6. Arazoe, H. et al. An autonomous actuator driven by fluctuations in ambient humidity. Nat. Mater. 15, 1084-1089 (2016).

7. Chen, J. et al. Artificial muscle-like function from hierarchical supramolecular assembly of photoresponsive molecular motors. Nat. Chem. 10, 132-138 (2018).
8. Cai, G., Ciou, J. H., Liu, Y., Jiang, Y. \& Lee, P. S. Leaf-inspired multiresponsive MXene-based actuator for programmable smart devices. Sci. Adv. 5, eaaw7956 (2019)

9. Yang, Y., Wu, Y., Li, C., Yang, X. \& Chen, W. Flexible actuators for soft robotics. Adv. Intell. Syst. 2, 1900077 (2019).

10. Nguyen, V. H. et al. Stimuli-responsive MXene-based actuators. Adv. Funct. Mater. 30, 1909504 (2020).

11. Wang, X. Q. et al. Somatosensory, light-driven, thin-film robots capable of integrated perception and motility. Adv. Mater. 32, e2000351 (2020).

12. Chen, S., Tan, M. W. M., Gong, X. \& Lee, P. S. Low-voltage soft actuators for interactive human-machine interfaces. Adv. Intell. Syst. 4, 2100075 (2021).

13. Wang, J., Gao, D. \& Lee, P. S. Recent progress in artificial muscles for interactive soft robotics. Adv. Mater. 33, e2003088 (2021).

14. Ilievski, F., Mazzeo, A. D., Shepherd, R. F., Chen, X. \& Whitesides, G. M. Soft robotics for chemists. Angew. Chem. Int. Ed. 50, 1890-1895 (2011).

15. Miriyev, A., Stack, K. \& Lipson, H. Soft material for soft actuators. Nat. Commun. 8, 596 (2017).

16. McCracken, J. M., Donovan, B. R. \& White, T. J. Materials as machines. $A d v$. Mater. 32, e1906564 (2020).

17. Mahato, M. et al. Sulfur- and nitrogen-rich porous $\pi$-conjugated COFs as stable electrode materials for electro-ionic soft actuators. Adv. Funct. Mater. 30, 2003863 (2020).

18. Roy, S., Kim, J., Kotal, M., Kim, K. J. \& Oh, I. K. Electroionic antagonistic muscles based on nitrogen-doped carbons derived from poly(triazinetriptycene). Adv. Sci. 4, 1700410 (2017).

19. Kim, O., Kim, S. J. \& Park, M. J. Low-voltage-driven soft actuators. Chem Commun. 54, 4895-4904 (2018).

20. Ma, S. et al. High-performance ionic-polymer-metal composite: toward largedeformation fast-response artificial muscles. Adv. Funct. Mater. 30, 1908508 (2019).

21. Kim, O., Kim, H., Choi, U. H. \& Park, M. J. One-volt-driven superfast polymer actuators based on single-ion conductors. Nat. Commun. 7, 13576 (2016).

22. Kim, O., Shin, T. J. \& Park, M. J. Fast low-voltage electroactive actuators using nanostructured polymer electrolytes. Nat. Commun. 4, 2208 (2013).

23. Kong, L. \& Chen, W. Carbon nanotube and graphene-based bioinspired electrochemical actuators. Adv. Mater. 26, 1025-1043 (2014).

24. Yan, Y. et al. Electroactive ionic soft actuators with monolithically integrated gold nanocomposite electrodes. Adv. Mater. 29, 1606109 (2017).

25. Acerce, M., Akdogan, E. K. \& Chhowalla, M. Metallic molybdenum disulfide nanosheet-based electrochemical actuators. Nature 549, 370-373 (2017).

26. Jo, C., Pugal, D., Oh, I.-K., Kim, K. J. \& Asaka, K. Recent advances in ionic polymer-metal composite actuators and their modeling and applications. Prog. Polym. Sci. 38, 1037-1066 (2013).

27. $\mathrm{Wu}, \mathrm{G}$. et al. Graphitic carbon nitride nanosheet electrode-based highperformance ionic actuator. Nat. Commun. 6, 7258 (2015).

28. Lu, C. et al. High-performance graphdiyne-based electrochemical actuators. Nat. Commun. 9, 752 (2018).

29. Roy, S. et al. Collectively exhaustive electrodes based on covalent organic framework and antagonistic Co-doping for electroactive ionic artificial muscles. Adv. Funct. Mater. 29, 1900161 (2019)

30. Kotal, M., Tabassian, R., Roy, S., Oh, S. \& Oh, I. K. Metal-organic frameworkderived graphitic nanoribbons anchored on graphene for electroionic artificial muscles. Adv. Funct. Mater. 30, 1910326 (2020).

31. Kotal, M., Kim, J., Kim, K. J. \& Oh, I. K. Sulfur and nitrogen Co-doped graphene electrodes for high-performance ionic artificial muscles. Adv. Mater. 28, 1610-1615 (2016).

32. Chen, I. P. et al. Newton output blocking force under low-voltage stimulation for carbon nanotube-electroactive polymer composite artificial muscles. ACS Appl. Mater. Interfaces 9, 5550-5555 (2017).

33. Cheedarala, R. K., Jeon, J.-H., Kee, C.-D. \& Oh, I.-K. Bio-inspired all-organic soft actuator based on a $\pi-\pi$ stacked $3 \mathrm{D}$ ionic network membrane and ultrafast solution processing. Adv. Funct. Mater. 24, 6005-6015 (2014)

34. Jeong, K. et al. Solvent-free, single lithium-ion conducting covalent organic frameworks. J. Am. Chem. Soc. 141, 5880-5885 (2019).

35. Ma, H. et al. Cationic covalent organic frameworks: A simple platform of anionic exchange for porosity tuning and proton conduction. J. Am. Chem. Soc. 138, 5897-5903 (2016).

36. Peng, Y. et al. Mechanoassisted synthesis of sulfonated covalent organic frameworks with high intrinsic proton conductivity. ACS Appl. Mater. Interfaces 8, 18505-18512 (2016).

37. Guo, Z. et al. Fast ion transport pathway provided by polyethylene glycol vonfined in covalent organic frameworks. J. Am. Chem. Soc. 141, 1923-1927 (2019).

38. Li, J. et al. A pre-synthetic strategy to construct single ion conductive covalent organic frameworks. Chem. Commun. 56, 2747-2750 (2020).

39. $\mathrm{Du}, \mathrm{Y}$. et al. Ionic covalent organic frameworks with spiroborate linkage. Angew. Chem. Int. Ed. 55, 1737-1741 (2016). 
40. Zhang, Y. et al. Three-dimensional anionic cyclodextrin-based covalent organic frameworks. Angew. Chem. Int. Ed. 56, 16313-16317 (2017).

41. Kong, Y. et al. Tight covalent organic framework membranes for efficient anion transport via molecular precursor engineering. Angew. Chem. Int. Ed. 60, 17638-17646 (2021).

42. Das, G. et al. Viologen-based conjugated covalent organic networks via zincke reaction. J. Am. Chem. Soc. 139, 9558-9565 (2017).

43. $\mathrm{Hu}, \mathrm{H}$. et al. Ionic covalent organic frameworks for highly effective catalysis. Chinese J. Catal. 39, 1437-1444 (2018).

44. Montoro, C. et al. Ionic conductivity and potential application for fuel cell of a modified imine-based covalent organic framework. J. Am. Chem. Soc. 139, 10079-10086 (2017)

45. Yu, F. et al. Photostimulus-responsive large-area two-dimensional covalent organic framework films. Angew. Chem. Int. Ed. 58, 16101-16104 (2019).

46. Yu, F. et al. Electrochromic two-dimensional covalent organic framework with a reversible dark-to-transparent switch. Nat. Commun. 11, 5534 (2020).

47. She, P., Qin, Y., Wang, X. \& Zhang, Q. Recent progress in external-stimulusresponsive 2D covalent organic frameworks. Adv. Mater. 33, e2101175 (2021).

48. Ying, Y. et al. Ultrathin two-dimensional membranes assembled by ionic covalent organic nanosheets with reduced apertures for gas separation. J. Am. Chem. Soc. 142, 4472-4480 (2020).

49. Sun, Q. et al. Reaction environment modification in covalent organic frameworks for catalytic performance enhancement. Angew. Chem. Int. Ed. 58, 8670-8675 (2019).

\section{Acknowledgements}

We acknowledge financial support from Singapore Ministry of Education Tier 1, RG63/ 20 (2020-T1-001-165) and the National Research Foundation, Prime Minister's Office, Singapore, under its Campus for Research Excellence and Technological Enterprise (CREATE) program.

\section{Author contributions}

P.S.L. supervised the project. P.S.L. and F.Y. conceived and designed the project. F.Y., J.H.C., S.H.C., W.C.P., J.C., J.T.C., K.H., J.L., and D.G. synthesized and characterized the materials. F.Y. conceived and built the device and contributed to the actuator device. F.Y. and P.S.L. analyzed the data and wrote the paper. All authors discussed the results and contributed to the paper.

\section{Competing interests}

The authors declare no competing interests.

\section{Additional information}

Supplementary information The online version contains supplementary material available at https://doi.org/10.1038/s41467-022-28023-2.

Correspondence and requests for materials should be addressed to Pooi See Lee.

Peer review information Nature Communications thanks Shengqian Ma and the other, anonymous, reviewer(s) for their contribution to the peer review of this work. Peer reviewer reports are available.

Reprints and permission information is available at http://www.nature.com/reprints

Publisher's note Springer Nature remains neutral with regard to jurisdictional claims in published maps and institutional affiliations.

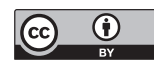

Open Access This article is licensed under a Creative Commons Attribution 4.0 International License, which permits use, sharing, adaptation, distribution and reproduction in any medium or format, as long as you give appropriate credit to the original author(s) and the source, provide a link to the Creative Commons license, and indicate if changes were made. The images or other third party material in this article are included in the article's Creative Commons license, unless indicated otherwise in a credit line to the material. If material is not included in the article's Creative Commons license and your intended use is not permitted by statutory regulation or exceeds the permitted use, you will need to obtain permission directly from the copyright holder. To view a copy of this license, visit http://creativecommons.org/ licenses/by/4.0/.

(C) The Author(s) 2022 